\title{
The short-term outcome of residual thrombus of the lower extremity after pharmacomechanical catheter-directed thrombolysis for deep vein thrombosis
}

\author{
Guang Chen ${ }^{1,2}$, Qing Wang ${ }^{2}$, Yahong Chen ${ }^{2}$, Xiang Wang ${ }^{2}$, Xiaolong Zhang ${ }^{2}$, Xiang $\mathrm{Li}^{2}$, Fei $\mathrm{Li}^{2}, \mathrm{Bin}^{\mathrm{Hu}}{ }^{2}$, \\ Haiwei $\mathrm{Chu}^{2}$, Dexing Zheng ${ }^{2}$, Wenjun Zhao ${ }^{2}$, Hongkun Zhang ${ }^{3}$ \\ ${ }^{1}$ Department of Vascular Surgery, The First Affiliated Hospital, College of Medicine, Zhejiang University, Hangzhou, China; ${ }^{2}$ Department of \\ Vascular Surgery, Taizhou Hospital of Zhejiang Province, Zhejiang University, Linhai, China; ${ }^{3}$ Department of Vascular Surgery, The First Affiliated \\ Hospital, College of Medicine, Zhejiang University, Hangzhou, China \\ Contributions: (I) Conception and design: G Chen, H Zhang; (II) Administrative support: W Zhao; (III) Provision of study materials or patients: G \\ Chen, W Zhao; (IV) Collection and assembly of data: Q Wang, Y Chen, X Wang, F Li, X Li, B Hu, H Chu, D Zheng, W Zhao; (V) Data analysis \\ and interpretation: G Chen, H Zhang; (VI) Manuscript writing: All authors; (VII) Final approval of manuscript: All authors. \\ Correspondence to: Hong-Kun Zhang. Department of Vascular Surgery, The First Affiliated Hospital, College of Medicine, Zhejiang University, \\ Hangzhou, China. Email: 1198050@zju.edu.cn.
}

Background: This study aimed to assess the short-term effect of residual thrombus of the lower extremity after pharmacomechanical catheter-directed thrombolysis (PCDT) on the incidence of post-thrombotic syndrome (PTS) and iliofemoral vein patency rate in patients who underwent PCDT.

Methods: There were 94 continuous patients with severe deep vein thrombosis (DVT) of the lower extremities admitted to our hospital between March 2016 and June 2018; 73 cases receiving PCDT and verified with iliac vein compression syndrome (IVCS) were assigned into two groups. Thirty-nine patients without thrombus of the popliteal and infrapopliteal veins were assigned to the proximal DVT group. The remaining 34 cases were placed in the extensive DVT group. The thrombus scores, including venous registry index (VRI) score, Marder score, and Society for Vascular Surgery (SVS) score before and after PCDT, the primary two years' cumulative rate of iliofemoral patency, and the rate of PTS, and complications were analyzed retrospectively.

Results: Ultimately, 27 patients in the proximal DVT group and 26 cases in the extensive DVT group completed the study. The two groups had no significant differences in terms of basic characteristics, complication and the rate of PTS $(\mathrm{P}>0.05)$. However, there were significant differences in terms of postoperative mean thrombus score and the mean degree of thrombosis removal score by Marder and SVS scores, residual thrombus by lower thrombosis classification (LET) and the two years' cumulative iliofemoral vein patency rate $(\mathrm{P}<0.05)$. And the logistic regression analysis demonstrated the residual thrombus of LET class II [odds ratio (OR), 4.619, 95\% confidence interval (CI), 1.090-19.567, P=0.038] was an independent risk factor for iliofemoral vein occlusion.

Conclusions: The residual thrombus of LET class II is an independent risk factor for iliofemoral vein occlusion. It is very important to keep the patency of the popliteal vein when deciding to use a stent to maintain iliofemoral vein patency. Furthermore, the anterior tibial vein approach and crisscross technique may be two important alternative methods that can be used to address the thrombus of popliteal vein for patients with extensive DVT.

Keywords: Deep vein thrombosis (DVT); pharmacomechanical catheter-directed thrombolysis (PCDT); postthrombotic syndrome (PTS); AngioJet

Submitted Jun 17, 2020. Accepted for publication Aug 10, 2020.

doi: $10.21037 / \mathrm{atm}-20-5459$

View this article at: http://dx.doi.org/10.21037/atm-20-5459 


\section{Introduction}

Anticoagulation is the global standard treatment for severe deep vein thrombosis (DVT) (1). However, anticoagulation does not remove the thrombus directly, and approximately one-third to one-half of severe proximal DVT cases will result in post-thrombotic syndrome (PTS) $(2,3)$. PTS is usually characterized by venous claudication, pain, fatigue, heaviness, limb swelling, and limb hyperpigmentation, and a few patients will develop severe acute manifestations like venous ulceration (4). Catheter-directed thrombolysis (CDT) is thought to be a safe procedure with an acceptable bleeding risk. In the CDT and Catheter-Directed Venous Thrombolysis in Severe Iliofemoral Vein Thrombosis (CaVenT) trial, CDT lowered the risks of the PTS for periods of 2 and 5 years, respectively $(5,6)$. Modern endovascular treatment options such as CDT, pharmacomechanical catheter-directed thrombolysis (PCDT) and stent placement have been used to lyse the thrombus, maintain venous valvular function and the patency of iliofemoral vein, and reduce the incidence of PTS. Nevertheless, in the Thrombus Removal with Adjunctive Catheter-Directed Thrombolysis (ATTRACT) trial, which comprised 194 cases of popliteal vein combined with proximal DVT with encouraged "infusion-first" therapy, CDT did not lower the risk of PTS compared to standard anticoagulation (7). Isolated calf DVT may be one of the main causes of the discrepancy between these trials. However, the rate of extension of DVT from calf DVT to proximal DVT across various studies is greatly variable due to the high heterogeneity in the population of patients, diagnostic strategies, and clinical settings $(8,9)$. Very little research has surveyed the effectiveness of pharmacomechanical CDT (PCDT) on treating proximal thrombosis combined with calf vein thrombosis. We consider the difference between these two trials, which may be related to the different residual thrombus positions of the enrolled patients. In this study, we aimed to evaluate effectiveness of the residual thrombus on the short-term iliofemoral vein patency rate and PTS rate after PCDT, and determine whether both extended DVT and proximal DVT patients could benefit from PCDT. We present the following article in accordance with the STROBE reporting checklist (available at http://dx.doi.org/10.21037/ atm-20-5459).

\section{Methods}

\section{Study design}

This was a retrospective study. The research protocol and waiver of informed consent were permitted by the institutional review board of our hospital. The study was conducted in accordance with the Declaration of Helsinki (as revised in 2013). The medical records of patients who had been admitted to our department from March 2016 to June 2018 with proximal DVT were reviewed, regardless of calf vein extension. Patients were excluded from the trial if they were older than 75 years old or younger than 14 years old, pregnant, allergic to urokinase, at high risk for bleeding, or hemodynamically unstable, or if they had symptoms for more than 14 days, contraindications to iodinated contrast media, active cancer, renal dysfunction, refractory hypertension, bacterial endocarditis, hyperthyroidism, or an aortic dissection or aneurysm. Patients who had PCDT and completed baseline and postoperative surveillance data were included in this research.And they were divided into proximal DVT group and extensive DVT group according to the residual thrombus of infra-popliteal vein after PCDT.

\section{Procedures}

All patients were started on low-molecular-weight heparin $(\mathrm{LMWH})$ as the initial anticoagulant prior to the surgical procedure. All procedures were performed in the hybrid endovascular operating theater. Patients were first placed in a supine position.

After application of a regional anesthetic, a 7-F sheath was put into the ipsilateral popliteal vein as directed by ultrasound to avoid punctures of nerves and artery walls. Patients were then placed in a prone position.

Inferior vena cava filters (Aegisy TM, Lifetech) were placed using the transfemoral technique. Unfractionated heparin boluses were provided in the process. Urokinase (Tianjin Biochemical Pharmaceutical, Tianjin, China) at a dose of $\leq 300,000$ units was delivered into the thrombus using the AngioJet Rheolytic Thrombectomy System (Boston Scientific). Then, 15-30 min after the initial delivery of urokinase, surgeons could use the AngioJet system to clear the thrombus. Venous registry index (VRI), Marder, and Society for Vascular Surgery (SVS) scores were collected before and after PCDT. The residual thrombosis and the obstructive lesions could be treated by adjunctive treatment, such as percutaneous transluminal balloon venoplasty, stent placement, or a combination with CDT. The model of adjunctive treatment was left to the discretion of the operative surgeon. The adjunctive treatment was disconnected when there was $90 \%$ thrombus removal with flow restoration or when an acute complication occurred. 
Rivaroxaban was recommended as the oral anticoagulant treatment for at least 3 months. In addition, graduated elastic compression stockings (ECSs) $(30-40 \mathrm{mmHg}$ ) were recommended for at least 2 years $(10,11)$. Clinical visits occurred at 10 and 30 days and at $3,6,9,12,15,18,21$, and 24 months by a surgeon and sonographer who were blinded to the treatment assignments.

\section{Primary efficacy outcome}

The growth of post-thrombosis syndrome was assessed by the Villalta scale, which is a clinical measure that integrates five subjective venous symptoms (pruritus, paresthesia, heaviness, cramps, and pain) and six objective venous signs (pain on calf compression, venous ectasia, redness, hyperpigmentation, skin induration, and pretibial edema) and the absence or presence of an ulcer in the leg (4). PTS was verified as a Villalta score of 5 or higher or an ulcer in the leg with an of index DVT occurring at any time between the 6-month and 24-month follow-up visit $(4,12)$.

\section{Secondary efficacy outcome}

The findings of compressibility of femoral vein, and flow in the pelvic and femoral vein by ultrasound were defined as no obstruction of functional vein flow and iliofemoral patency (7). The scores of thrombus, SVS, Marder, and VRI were evaluated before and after $\operatorname{PCDT}(13,14)$.

\section{Safety outcomes}

Safety outcomes were assessed by monitoring bleeding, acute kidney injury (AKI), recurrent venous thromboembolism, and death. Outcomes from day 10 to month 24 were record and summarized (15).

\section{Statistical analysis}

Data from proximal DVT and extensive DVT groups were compared. Statistical analyses were conducted utilizing 11.6 version MedCalc (MedCalc Software 2011, Mariakerke, Belgium), 20.0 SPSS software for Windows (IBM Corp, Armonk, NY, USA) and GraphPad Prism 7 software (GraphPad Software Inc., La Jolla, CA, USA).

Continuous variables with normal distribution were reported as the mean \pm standard deviation $(\mathrm{SD})$ and compared by two-tailed independent $t$-test. Continuous variables with non-normal distribution are presented as the median (interquartile range) and were compared using the Mann-Whitney $\mathrm{U}$ test. Categorial data were compared by $\chi^{2}$ test. If a cell value was less than 5 , it was compared by Fisher's exact test. Patients' primary iliofemoral vein patency rate and PTS cumulative incidence within 24 months among the two groups was compared by using KaplanMeier survival analysis. Univariable regression analysis and multivariate regression analysis were used to evaluate risk factors of iliofemoral vein patency and PTS. A P value $<0.05$ was considered to indicate statistical significance.

\section{Results}

\section{Features of the patients at baseline}

From March 2016 through June 2018, 94 consecutive patients confirmed with acute lower extremity DVT by ultrasound were admitted to our hospital. Three patients received anticoagulation therapy only, while the remaining 91 cases were treated by PCDT. After the treatment with PCDT, 17 cases without iliac vein compression syndrome (IVCS) and 1 case with bilateral DVT were excluded from the trial. The remaining 73 cases confirmed with IVCS were divided into two groups. Of these, 39 patients without residual popliteal and infrapopliteal vein thrombus were placed in the proximal DVT group, and the remaining 34 cases with residual popliteal vein thrombus or concomitant calf vein thrombosis were assigned to the extensive DVT group. Three patients died and nine were lost to follow-up in the proximal DVT group, while no patients died and eight were lost to follow-up in the extensive DVT group (Figure 1).

Patients' clinical features and baseline demographics are provided in Table 1. Ten patients in the proximal DVT group versus three patients in the extensive DVT group had a history of major surgery, and the difference was significant $(\mathrm{P}=0.031)$. Meanwhile the there was no significance differences between these two groups in median age, median interval from the onset of symptoms, location of thrombosis, and concomitant comorbidities.

There were no significant differences in the additional treatment, the number of stent placements, the relationship of stent and the inguinal ligament, and the complication rate after PCDT in these two groups $(\mathrm{P}>0.05)$. When comparing the residual thrombus of thrombosis classification (LET) class I and LET class II, however, the two groups showed significant differences $(\mathrm{P}<0.05)$ (Table 2).

The preprocedure and postprocedure thrombosis scores including VRI, Marder, and SVS score were calculated. 


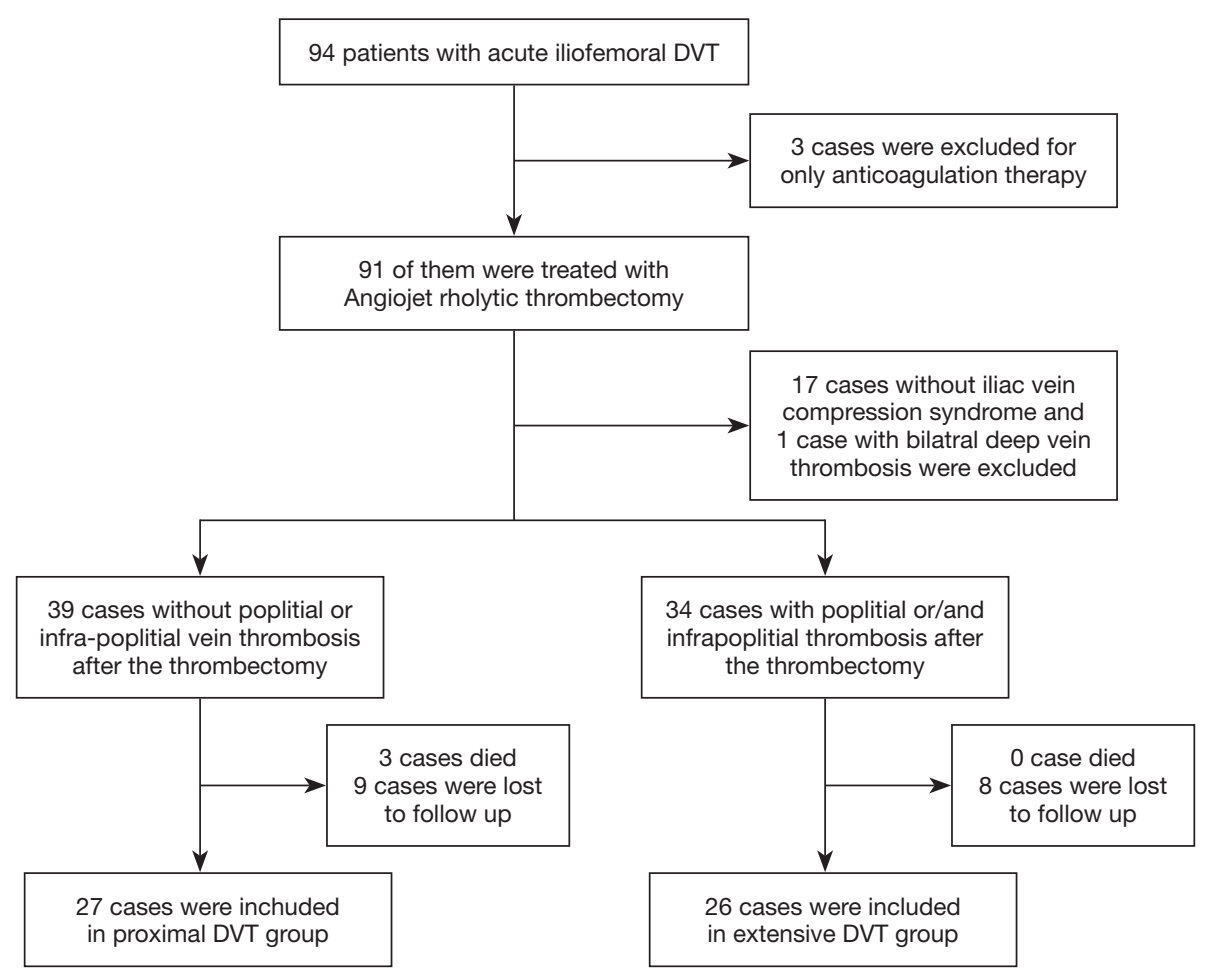

Figure 1 Enrollment and. DVT, deep vein thrombosis.

Table 1 Patient characteristics at baseline

\begin{tabular}{|c|c|c|c|}
\hline Baseline & Proximal DVT group $(\mathrm{n}=27)$ & Extensive DVT group $(n=26)$ & $P$ value \\
\hline Median age [IQR], yr" & $67[56-73]$ & $66.5[53-75]$ & 0.889 \\
\hline Male, No. [\%] & 9 [37] & $7[27]$ & 0.611 \\
\hline The median interval from the onset of symptoms [IQR], days" & $4[2-6]$ & $5[3-10]$ & 0.131 \\
\hline The location of deep vein thrombosis, No. [\%] $]^{\top}$ & & & 1.000 \\
\hline Right-sided DVT & $3[11]$ & $2[8]$ & \\
\hline History of previous DVT, No. [\%] $]^{\top}$ & $2[7]$ & 3 [12] & 0.669 \\
\hline Major surgery, No. $[\%]^{f \neq}$ & $10[37]$ & $3[12]$ & 0.031 \\
\hline
\end{tabular}

There were significant differences in these two groups in relation to preprocedure thrombosis scores $(\mathrm{P}<0.01)$. However, no evident differences in these two groups were found when comparing postprocedure thrombosis score and the mean degree of thrombosis removal by VRI score $(\mathrm{P}>0.05)$. The mean degree of thrombosis removal in these groups, including the VRI, Marder, and SVS scores were $70.00 \% \pm 28.66 \%$ vs. $76.51 \% \pm 12.13 \% \quad(\mathrm{P}=0.286)$,
77.74\% $\pm 17.19 \%$ vs. $53.15 \% \pm 16.89 \%(\mathrm{P}<0.001)$, and $83.86 \% \pm 11.65 \%$ vs. $66.59 \% \pm 13.69 \%(\mathrm{P}<0.001)$, respectively (Table 3).

\section{PTS and iliofemoral venous patency}

There were significant differences in the cumulative iliofemoral vein patency rates at 24 months and average 
Table 2 Details of treatment and complication

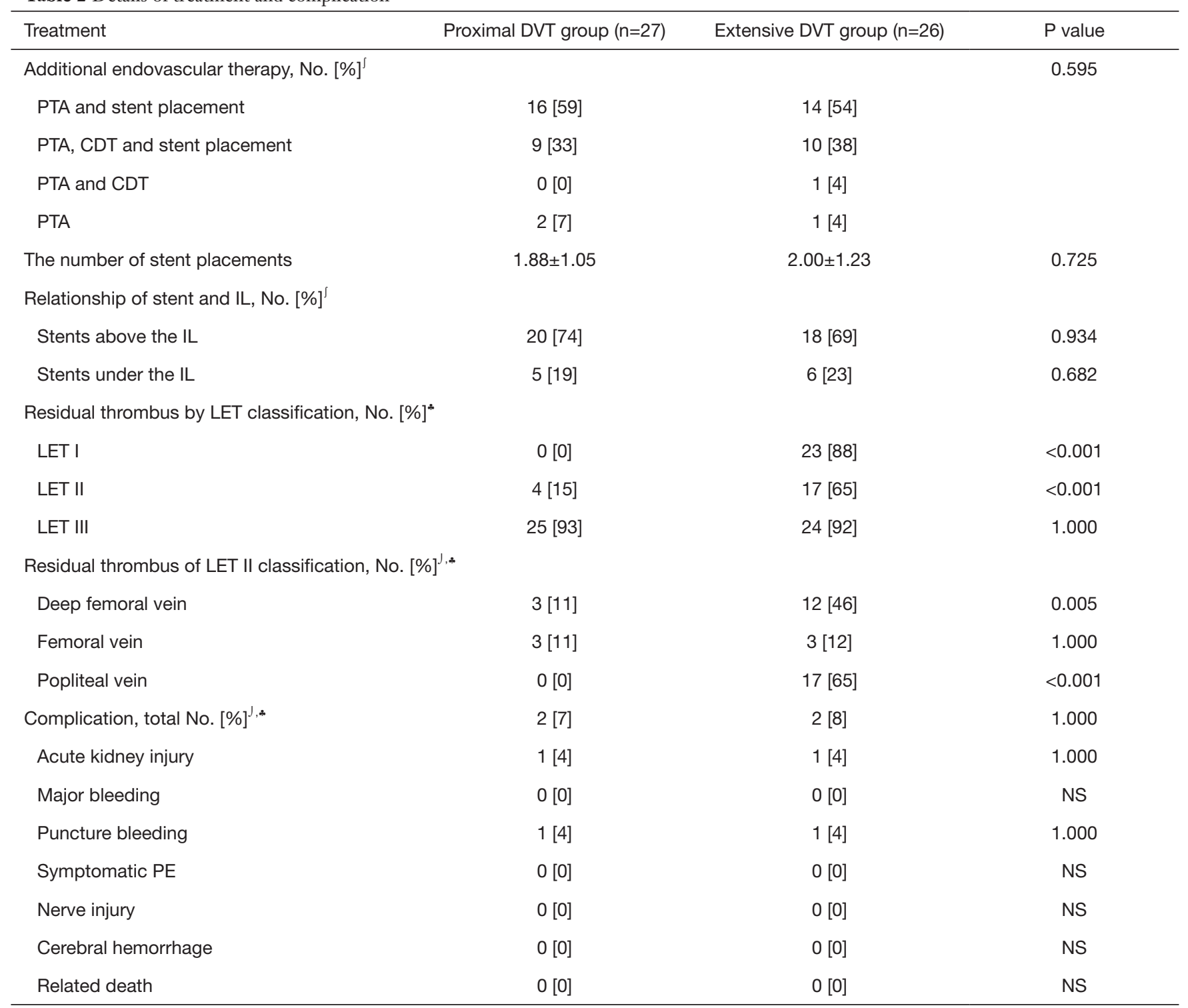

', data are No. [\%]; ' , LET classification including four classes; LET I includes calf vein thrombosis; LET II includes the popliteal, distal femoral vein, proximal femoral vein, and deep femoral vein; LET III includes the common femoral vein, external iliac vein, and common iliac vein; LET IV includes the infra-renal inferior vena cava and supra-renal inferior vena cava; *, patients could be included in more than one category. CIV, common iliac vein; EIV, external iliac vein; CFV, common femoral vein; SFV, superficial femoral vein; LT, lesser trochanter; IL, inguinal ligament.

Villatla scores at 6 months between these two groups (Table 4, Figure 2). However, there were no significant differences in the rate of oral anticoagulation drug intake, wearing ECSs, and the cumulative PTS rate at 6 months and 24 months or in the average Villatla score at 24 months $(\mathrm{P}>0.05)$ (Table 4, Figure 3). The univariable logistic regression and the multivariable logistic regression analyses indicated that the residual thrombus of LET II [odds ratio (OR), 4.619, $95 \%$ confidence interval (CI), 1.090-19.567, $\mathrm{P}=0.038]$ was an independent risk factor for raising iliofemoral vein occlusion by LET classification (Figures 4,5). Despite this, univariable logistic regression and the multivariable logistic regression analyses did not find any independent risk factor for the PTS when using residual 
Table 3 Preoperative and postoperative thrombotic scores

\begin{tabular}{|c|c|c|c|}
\hline Thrombotic scores & Proximal DVT group $(n=27)$ & Extensive DVT group $(n=27)$ & $P$ value \\
\hline $\mathrm{VRI}^{\mathrm{J}}$ & $7.48 \pm 2.58$ & $11.35 \pm 1.57^{\dagger}$ & $<0.001$ \\
\hline Marde $e^{r f}$ & $15.33 \pm 7.81$ & $34.62 \pm 7.91^{\dagger}$ & $<0.001$ \\
\hline NA-ISCVS/SVS" & $7.22 \pm 2.33$ & $13.42 \pm 2.39^{\dagger}$ & $<0.001$ \\
\hline \multicolumn{4}{|l|}{ Postoperative score } \\
\hline $\mathrm{VRI}^{\prime}$ & $2.26 \pm 2.18$ & $2.62 \pm 1.27$ & 0.469 \\
\hline Marder ${ }^{\jmath}$ & $3.33 \pm 2.50$ & $16.96 \pm 7.57^{\dagger}$ & $<0.001$ \\
\hline NA-ISCVS/SVS" & $1.19 \pm 0.83$ & $4.50 \pm 1.79^{\dagger}$ & $<0.001$ \\
\hline \multicolumn{4}{|c|}{ Difference before and after PMT } \\
\hline NA-ISCVS/SVS" & $6.04 \pm 2.12$ & $8.92 \pm 2.23^{\dagger}$ & $<0.001$ \\
\hline \multicolumn{4}{|c|}{ The mean degree of thrombosis removal } \\
\hline $\mathrm{VRI}^{J}$ & $70.00 \pm 28.66$ & $76.51 \pm 12.13$ & 0.286 \\
\hline Marder $^{\jmath}$ & $77.74 \pm 17.19$ & $53.15 \pm 16.89^{\dagger}$ & $<0.001$ \\
\hline NA-ISCVS/SVS" & $83.86 \pm 11.65$ & $66.59 \pm 13.69^{\dagger}$ & $<0.001$ \\
\hline
\end{tabular}

J, VRI scoring system included 7 venous segments, with each segment being worth 2 points for a total score of 14 points. The criteria for scoring are classified as follows: completely free of thrombus =0; partially occluded =1; and completely occluded =2; ', Marder score ranges from 0 to 24, with 0 representing no thrombus and 24 representing complete thrombosis; ", the NA-ISCVS/SVS reporting standards including 6 deep and the 2 superficial venous segments, with each segment being assigned the following scores: 0 for a patent segment, 1 for a subsegmental nonocclusive thrombus, 2 for a subsegmental occlusive thrombus, and 3 for an occlusive thrombus throughout the length of a segment; ${ }^{\dagger}$, continuous variables were compared with $\chi^{2}$ test; $\mathrm{P}<0.05$. Data are mean $\pm \mathrm{SD}$. VRI, venous registry index; SVS, Society for Vascular Surgery.

thrombus by LET classification.

\section{Safety outcomes}

All 73 patients exhibited hematuria after PCDT. Two patients developed AKI after PCDT, and required short-term dialysis before discharge, while two patients experienced puncture site bleeding. However, there were no major bleeding events, deaths related to PCDT treatment, cerebral hemorrhage, nerve injury, or symptomatic $\mathrm{PE}$ found in either group. These two groups had no significant differences in terms of adverse events $(\mathrm{P}=1.000$, Table 2).

\section{Discussion}

In this trial, there were no significant differences in the incidence of the PTS between these two groups at 6 and
24 months $(P>0.05)$. However, when compared with the CDT group in the CaVenT study, and the pharmacomechanical thrombolysis (PMT) group in the ATTRACT trial, the total cumulative PTS incidence was lower than those in the Caven T and ATRRACT trials at 6and 24-month follow-up $(\mathrm{P}<0.05)(6,15)$ (Table 5).

These differences might be related to several factors. First, all of the patients assigned to this trial were verified with IVCS by venography. Except for 4 cases without obvious residual thrombus after PCDT, all cases [69] were treated with stent placement to reduce the high recurrence rate of thrombosis and maintain the high patency rate of the iliofemoral vein, whereas only $16.67 \%$ patients in the CaVenT study and $24.40 \%$ of patients in the ATTRACT trial received stent placement $(\mathrm{P}<0.05)(16)$. Because stent placement was encouraged only to obtain lower than $50 \%$ of residual stenosis in the CavenT study, or with lesions 
Table 4 The patients' compliance and short-term outcome of residual thrombus

\begin{tabular}{|c|c|c|c|}
\hline Compliance and outcome & Proximal DVT group $(n=27)$ & Extensive DVT group $(n=26)$ & $P$ value \\
\hline Oral anticoagulation at 24 months, No. [\%] & $11[41]$ & $8[(31]$ & 0.449 \\
\hline Wear with ECS at 6 months, No. [\%] & $20[75]$ & 19 [74] & 0.934 \\
\hline Wear with ECS at 24 months, No. [\%] & $15[56]$ & $15[58]$ & 0.875 \\
\hline The mean Villalta score at 24 months $^{\jmath}$ & $1.41 \pm 1.93$ & $2.38 \pm 1.98$ & 0.075 \\
\hline PTS at 6 months, No. [\%] $]^{\jmath}$ & $1[4]$ & $4[15]$ & 0.192 \\
\hline PTS at 24 months, No. [\%] $]^{\jmath}$ & $3[11]$ & $4[15]$ & 0.704 \\
\hline Iliofemoral patency at 6 months, No. [\%] & 25 [93] & 22 [85] & 0.420 \\
\hline
\end{tabular}

', the Villalta scale incorporates the assessment of 5 subjective venous symptoms and 6 objective venous signs, as well as the presence or absence of ulcers; a the total score more than 5 indicates post-thrombotic syndrome; ' , lliofemoral patency was defined as regained when the following findings were present: flow in the pelvic and femoral vein, compressibility of the femoral vein, and no functional venous obstruction at any level; ${ }^{\dagger}$, continuous variables were compared with $\chi^{2}$ test, $\mathrm{P}<0.05 ;{ }^{\ddagger}$, binary variables were compared with $\chi^{2}$ test, $\mathrm{P}<0.05$. Data are No. [\%]. ECS, elastic compression stocking; PTS, post-thrombotic syndrome.

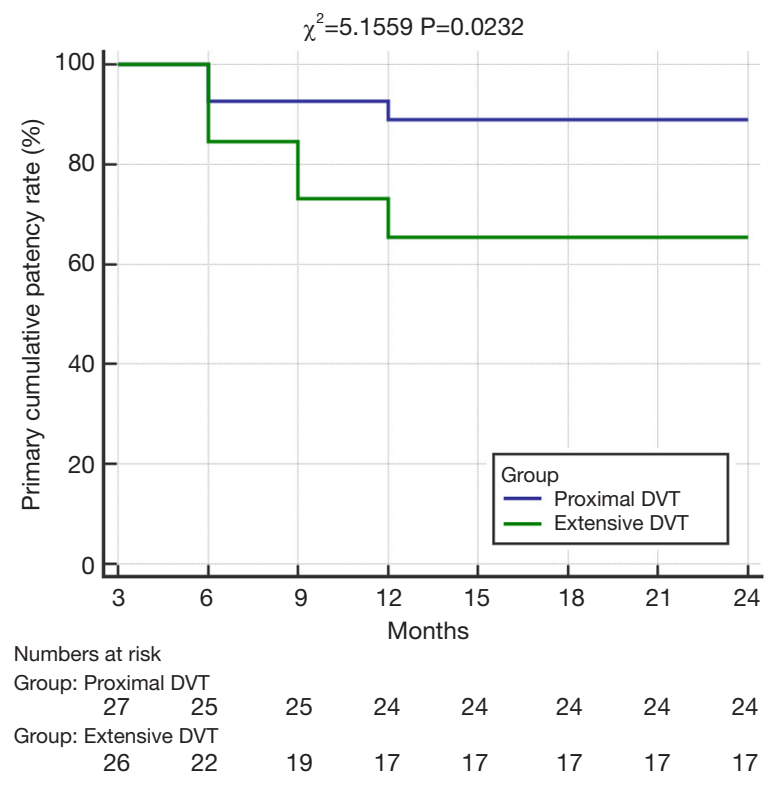

Figure 2 Primary cumulative patency rate. Shown is the incidence of iliofemoral vein cumulative patency of patients assigned to proximal DVT group and those assigned to the extensive DVT group. Values for incidence were calculated with the use of KaplanMeier methods and were compared with the use of the log-rank test. DVT, deep vein thrombosis.

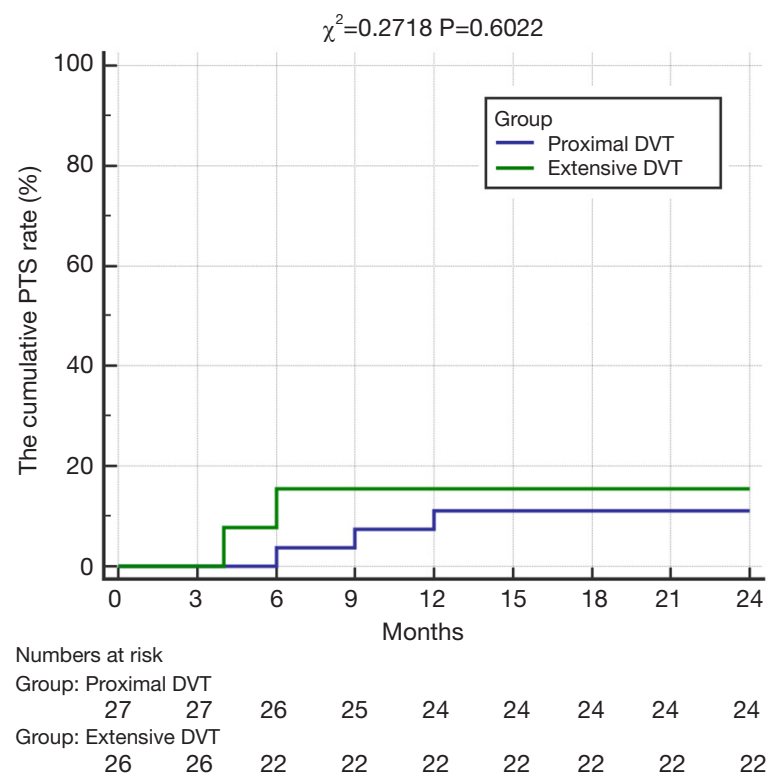

Figure 3 Cumulative post-thrombotic syndrome rate. Shown is the incidence of post-thrombotic syndrome of patients assigned to proximal DVT group and those assigned to the extensive DVT group. Values for incidence were calculated with the use of KaplanMeier methods and were compared with the use of the log-rank test. DVT, deep vein thrombosis. 


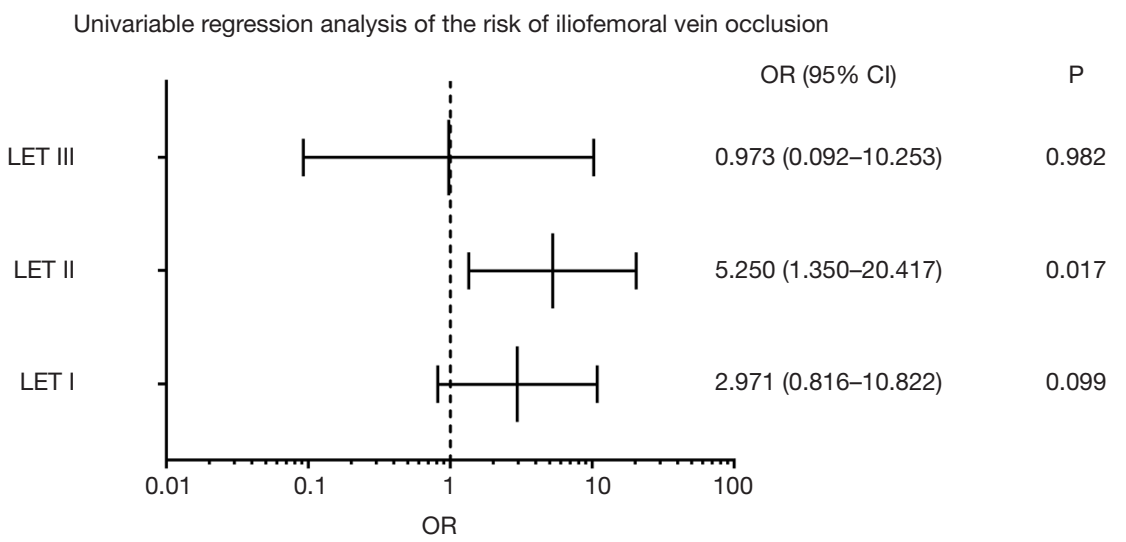

Figure 4 OR of the risk of iliofemoral vein occlusion. Shown is the odds ratio of the risk of iliofemoral vein occlusion among patients with residual thrombus by univariable regression analysis. OR, odds ratio; LET, lower thrombotic classification.

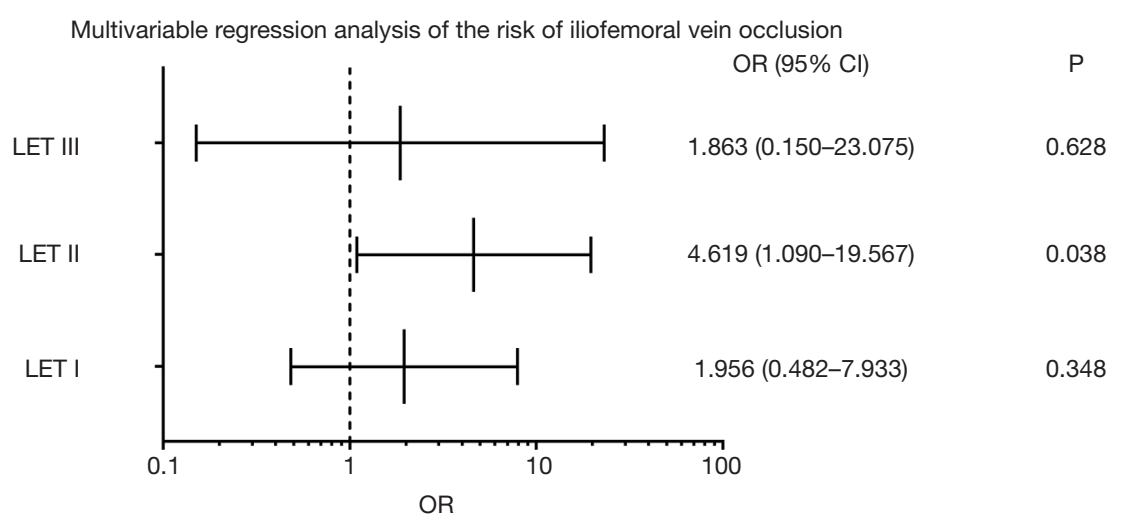

Figure 5 OR of the risk of iliofemoral vein occlusion. Shown is the odds ratio of the risk of iliofemoral vein occlusion among patients with residual thrombus by multivariable regression analysis. OR, odds ratio; LET, lower thrombotic classification.

causing more than $50 \%$ stenosis, robus collateral filling or a mean pressure gradient was more than $2 \mathrm{mmHg}$ in the ATTRACT trial $(6,17)$. The differences in the criteria of stent placement might be one of the important reasons for the differences among these trials. Second, the compliance rate of patients on oral anticoagulation was significantly different among these three trials $(\mathrm{P}<0.05)$. In this trial, $94.34 \%$ patients were still on oral anticoagulation at 6 months follow-up, whereas the rate was $78.28 \%$ in the ATTRACT trial and $61.11 \%$ in the CaVenT study. It is commonly accepted that the risks of recurrent venous thromboebolism could be substantially decreased through an adequate course of anticoagulation therapy $(18,19)$. The difference in compliance may be another important reason for differences among these three trials. Third, $88.68 \%$ patients in this trial were confirmed with leftsided vein thrombosis, whereas the rate was $60.00 \%$ in the CaVenT study, and $58.03 \%$ in the ATRRACT trial $(\mathrm{P}<0.05)$. However, the proportion of left lower extremity DVT between these two groups was not significantly different $(\mathrm{P}>0.05)$, and any difference in the proportion might have an effect on the rate of PTS. Fourth, the type of ECS might play an important role in the PTS. Though the Sox trial and two previous RCTs used different evaluations for DVT treatment, the graduated ECSs could help the pump the calf muscle and lower reflux and venous hypertension, thereby enhancing the microcirculation of tissue and lowering edema $(10,11,20,21)$. Although no significant differences 
Table 5 Comparison with the CaVenT trial and the ATTRACT trial

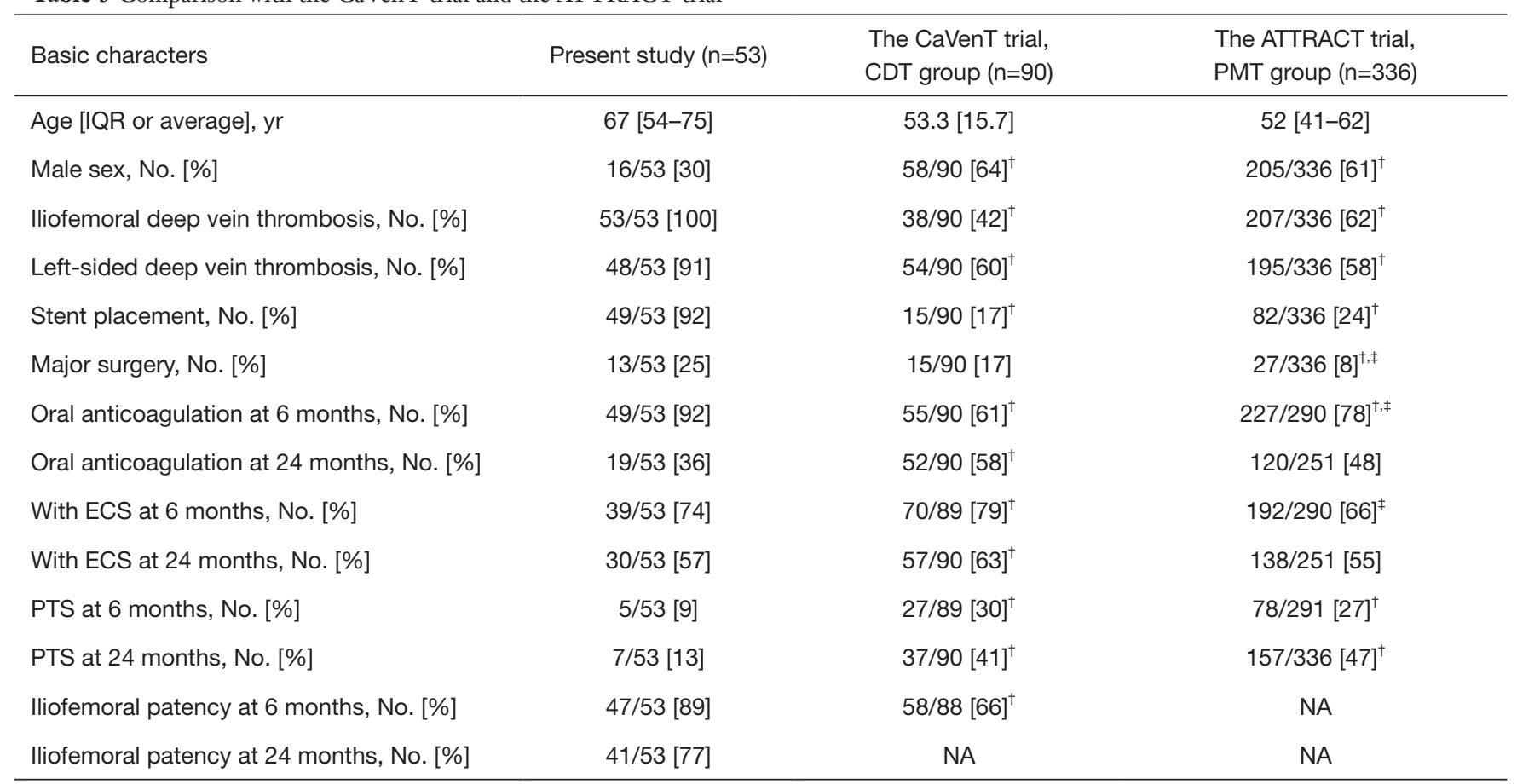

${ }^{\dagger}$, compared with this trial using $\chi^{2}$ test, $\mathrm{P}<0.05 .{ }^{\ddagger}$, compared between the CDT group and PMT group using $\chi^{2}$ test, $\mathrm{P}<0.05$. IQR denotes interquartile range. Data are No./total No. [\%]. CDT, catheter-directed thrombolysis; PMT, pharmacomechanical-thrombolysis; ECS, elastic compression stockings.

were found in the rate of wearing ECSs at 24-month followup in these three trials $(\mathrm{P}>0.05)$, all patients in the present study received compression therapy with above-knee thighlength ECSs, whereas patients in the CaVenT trial and the ATTRACT trial were treated with knee-high ECSs. We speculate that the different types of ECSs might be a reason for the difference in effectiveness for preventing PTS.

The cumulative iliofemoral vein patency rate at 2 years was $88.46 \%$ in the proximal DVT group and $62.96 \%$ in the extensive group, which represents a significant difference $(\mathrm{P}<0.05)$. Except for the preoperative and postoperative thrombotic scores via Marder score and SVS score, there were no significant differences in the basic characteristics and additional endovascular therapies between these two groups. For the residual thrombus after PCDT leads to stenosis of the inflow tract, IVCS leads to the restriction of the outflow tract blood flow, and the lack of anticoagulant therapy are risk factors for iliac vein occlusion. We speculate the residual thrombus after PCDT might play an important role in maintaining the iliofemoral vein patency and the development of PTS.

Univariable regression analysis did not demonstrate any
LET class of residual thrombus to be an independent risk factors for PTS in these two groups while using the LET classification (22). This in line with the study by Jeyabalan et al. which reported that inflow thrombosis did not affect PTS development (23).

In contrast, univariable and multivariable regression analyses did demonstrate the residual thrombus of LETII (OR, 4.619, 95\% CI, 1.090-19.567, $\mathrm{P}=0.038$ ) to be an independent risk factor for iliofemoral vein occlusion. It is thus crucial to clear the thrombus of LET II before stent placement for those cases with extended DVT, as the popliteal vein is an important part of LET II classification. In order to clear all the thrombi of LET II, it is important to clear the thrombus of the popliteal vein. However, traditional PCDT, including CDT, usually uses the popliteal vein for access, and it cannot the thrombus of the infrapopliteal vein cannot be cleared $(24,25)$. In order to address the issue, Wang et al. demonstrated that CDT via an anterior approach of the tibial vein was a safe, effective, and feasible method for patients with severe extended lower extremity DVT. Indeed, the patency rate of $83.51 \%$ (81/97) in their study appears superior to that reported in 
the CaVenT trial (26). Rego et al. suggested that the crisscross technique as another alternative method to solve thrombus of the infrapopliteal vein; this technique uses retrograde vascular access and simultaneous antegrade ("criss-cross") access to the popliteal vein to accomplish recanalization of the vein in patients with acute iliofemoral DVT and concomitant infrapopliteal vein thrombosis (27). In the CaVenT trial, only patients confirmed with DVT above the mid-thigh level were included for study (6). In the ATTRACT trial, however, patients with thrombosis extended to the popliteal vein and or infrapopliteal vein were included (17). In our study, only 4 cases in the proximal DVT group and 17 cases in the extensive DVT group had residual thrombus of LETII after PCDT, which is a significant difference $(\mathrm{P}<0.05)$. We can thus speculate that different criteria for defining the range of thrombosis might reflect different residual thrombus evaluations of LETII after CDT or PCDT in these two trials, and that this may be a neglected cause which contributed to the discrepancies of these two trials. We hope larger, randomized controlled trials can explore the feasibility of the two alternative approaches and the influence of residual thrombus after CDT or PCDT on ilofemoral vein patency and the development of PTS in patients with severe thrombosis of the lower extremities.

In our previous research, we found that severe hemolysis resulting from PMT with AngioJet (AJ-PMT) and a history of surgery at 3 months were independent risk factors for the growth of AKI (28). Consequently, we have limited the aspiration time and volumes of PCDT, and have used sodium bicarbonate and hydration to protect kidney function. There were only two cases of AKI after PCDT in the proximal DVT group and the extend DVT group, and no significant differences were found in the complications between these two groups $(\mathrm{P}>0.05)$.

Some limitations to our research should be addressed. First, it was a single-centered retrospective study with a small sample size and a high rate of cases lost to follow-up. Thus, the statistical power was limited, and type II errors might have arisen. Second, the male-to-female ration and the rate of stent placement were different from those of the CaVenT and ATTRACT trials. As a consequence, the result might not be generalizable to patients with DVT. Third, large randomized controlled trials are needed to explain the influence of residual thrombus on iliofemoral vein patency and the development of PTS, and to determine whether or not the alternative methods that use the infrapopliteal vein approach or the criss-cross technique are superior to the traditional popliteal vein approach in treating acute lower extremity DVT involving the popliteal vein and/or infrapopliteal vein.

\section{Conclusions}

Our results demonstrate that the residual thrombus of LET II increases the chance of iliofemoral vein occlusion in cases with severe lower extremity DVT. As the conventional approach by popliteal vein cannot clear the thrombus below the puncture site of popliteal vein and the infrapopliteal vein, the anterior tibial vein approach or the criss-cross technique may be two important alternative methods to the conventional popliteal approach during PCDT. This may also partly explain the inconsistencies between the CaVenT and ATRRACT trials. We believe that the results of our present research represent an important supplement to the information regarding the treatment of severe lower extremity DVT.

\section{Acknowledgments}

Funding: The sponsor of the trial was Taizhou Hospital affiliated to Zhejiang University. We thank all the patients and collaborators who participated or contributed to the study. The study was financially supported by grants from the Taizhou Municipal Bureau of Science and Technology (No. 1901KY09) and the Zhejiang Province Public Welfare Application Technology Research Plan (grant No. LGF20H020007).

\section{Footnote}

Reporting Checklist: The authors have completed the STROBE reporting checklist. Available at http://dx.doi. org/10.21037/atm-20-5459

Data Sharing Statement: Available at http://dx.doi. org/10.21037/atm-20-5459

Conflicts of Interest: All authors have completed the ICMJE uniform disclosure form (available at http://dx.doi. org/10.21037/atm-20-5459). The authors have no conflicts of interest to declare.

Ethical Statement: The authors are accountable for all aspects of the work in ensuring that questions related to the accuracy or integrity of any part of the work are 
appropriately investigated and resolved. This was a retrospective study. The research protocol and waiver of informed consent were permitted by the institutional review board of our hospital. The study was conducted in accordance with the Declaration of Helsinki (as revised in 2013).

Open Access Statement: This is an Open Access article distributed in accordance with the Creative Commons Attribution-NonCommercial-NoDerivs 4.0 International License (CC BY-NC-ND 4.0), which permits the noncommercial replication and distribution of the article with the strict proviso that no changes or edits are made and the original work is properly cited (including links to both the formal publication through the relevant DOI and the license). See: https://creativecommons.org/licenses/by-nc-nd/4.0/.

\section{References}

1. Kearon C, Akl EA, Comerota AJ, et al. Antithrombotic therapy for VTE disease: Antithrombotic Therapy and Prevention of Thrombosis, 9th ed: American College of Chest Physicians Evidence-Based Clinical Practice Guidelines. Chest 2012;141:e419S-96S.

2. van Dongen CJ, Prandoni P, Frulla M, et al. Relation between quality of anticoagulant treatment and the development of the postthrombotic syndrome. J Thromb Haemost 2005;3:939-42.

3. Cosmi B, Palareti G. "Early thrombus removal" in iliacfemoral deep vein thrombosis for prevention of postthrombotic syndrome. Ann Transl Med 2019; 7:S343.

4. Kahn SR, Partsch H, Vedantham S, et al. Definition of post-thrombotic syndrome of the leg for use in clinical investigations: a recommendation for standardization. J Thromb Haemost 2009;7:879-83.

5. Haig Y, Enden T, Grotta O, et al. Post-thrombotic syndrome after catheter-directed thrombolysis for deep vein thrombosis (CaVenT): 5-year follow-up results of an open-label, randomised controlled trial. Lancet Haematol 2016;3:e64-71.

6. Enden T, Haig Y, Klow NE, et al. Long-term outcome after additional catheter-directed thrombolysis versus standard treatment for acute iliofemoral deep vein thrombosis (the CaVen T study): a randomised controlled trial. Lancet 2012;379:31-8.

7. Enden T, Sandvik L, Klow NE, et al. Catheterdirected Venous Thrombolysis in acute iliofemoral vein thrombosis--the CaVenT study: rationale and design of a multicenter, randomized, controlled, clinical trial (NCT00251771). Am Heart J 2007;154:808-14.

8. Righini M, Paris S, Le Gal G, et al. Clinical relevance of distal deep vein thrombosis. Review of literature data. Thromb Haemost 2006;95:56-64.

9. Garry J, Duke A, Labropoulos N. Systematic review of the complications following isolated calf deep vein thrombosis. Br J Surg 2016;103:789-96.

10. Prandoni P, Lensing AW, Prins MH, et al. Belowknee elastic compression stockings to prevent the postthrombotic syndrome: a randomized, controlled trial. Ann Intern Med 2004;141:249-56.

11. Brandjes DP, Buller HR, Heijboer H, et al. Randomised trial of effect of compression stockings in patients with symptomatic proximal-vein thrombosis. Lancet 1997;349:759-62.

12. Kahn SR. Measurement properties of the Villalta scale to define and classify the severity of the post-thrombotic syndrome. J Thromb Haemost 2009;7:884-8.

13. Porter JM, Moneta GL. Reporting standards in venous disease: an update. International Consensus Committee on Chronic Venous Disease. J Vasc Surg 1995;21:635-45.

14. Marder VJ, Soulen RL, Atichartakarn V, et al. Quantitative venographic assessment of deep vein thrombosis in the evaluation of streptokinase and heparin therapy. J Lab Clin Med 1977;89:1018-29.

15. Schulman S, Kearon C, Subcommittee on Control of Anticoagulation of the $\mathrm{S}$, et al. Definition of major bleeding in clinical investigations of antihemostatic medicinal products in non-surgical patients. J Thromb Haemost 2005;3:692-4.

16. Husmann MJ, Heller G, Kalka C, et al. Stenting of common iliac vein obstructions combined with regional thrombolysis and thrombectomy in acute deep vein thrombosis. Eur J Vasc Endovasc Surg 2007;34:87-91.

17. Vedantham S, Goldhaber SZ, Julian JA, et al. Pharmacomechanical Catheter-Directed Thrombolysis for Deep-vein thrombosis. N Engl J Med 2017;377:2240-52.

18. Kearon C, Hirsh J. Management of anticoagulation before and after elective surgery. N Engl J Med 1997;336:1506-11.

19. Gonzalez-Fajardo JA, Arreba E, Castrodeza J, et al. Venographic comparison of subcutaneous low-molecular weight heparin with oral anticoagulant therapy in the long-term treatment of deep venous thrombosis. J Vasc Surg 1999;30:283-92.

20. Kahn SR, Elman E, Rodger MA, et al. Use of elastic compression stockings after deep venous thrombosis: a 
comparison of practices and perceptions of thrombosis physicians and patients. J Thromb Haemost 2003;1:500-6.

21. Kahn SR, Shapiro S, Wells PS, et al. Compression stockings to prevent post-thrombotic syndrome: a randomised placebo-controlled trial. Lancet 2014;383:880-8.

22. Strijkers RH, Arnoldussen CW, Wittens CH. Validation of the LET classification. Phlebology 2015;30:14-9.

23. Jeyabalan G, Marone L, Rhee R, et al. Inflow thrombosis does not adversely affect thrombolysis outcomes of symptomatic iliofemoral deep vein thrombosis. J Vasc Surg 2011;54:448-53.

24. Duan PF, Ni CF. Randomized study of different approaches for catheter-directed thrombolysis for lowerextremity acute deep venous thrombosis. J Formos Med Assoc 2016;115:652-7.

25. Liu G, Liu X, Wang R, et al. Catheter-Directed Thrombolysis of Acute Entire Limb Deep Vein

Cite this article as: Chen G, Wang Q, Chen Y, Wang X, Zhang X, Li X, Li F, Hu B, Chu H, Zheng D, Zhao W, Zhang H. The short-term outcome of residual thrombus of the lower extremity after pharmacomechanical catheter-directed thrombolysis for deep vein thrombosis. Ann Transl Med 2020;8(16):1001. doi: 10.21037/atm-20-5459
Thrombosis From below the Knee Access: A Retrospective Analysis of a Single-Center Experience. Catheter Cardiovasc Interv 2018;91:310-7.

26. Wang H, Qi X, Luo H, et al. Catheter-directed thrombolysis through anterior tibial vein for treating acute extensive deep venous thrombosis. J Vasc Surg Venous Lymphat Disord 2018;6:681-8.

27. Rego DC, Ahmed AKS, O'Sullivan GJ. Addressing Infrapopliteal Thrombus in Iliofemoral DVT: The Criss-Cross Technique. Cardiovasc Intervent Radiol 2018;41:1095-9.

28. Shen Y, Wang X, Jin SS, et al. Increased risk of acute kidney injury with percutaneous mechanical thrombectomy using AngioJet compared with catheterdirected thrombolysis. J Vasc Surg Venous Lymphat Disord 2019;7:29-37.

(English Language Editor: J. Gray) 